\title{
Picosecond magnetization dynamics in nanomagnets: Crossover to nonuniform precession
}

\author{
V. V. Kruglyak,* A. Barman, and R. J. Hicken ${ }^{\dagger}$ \\ School of Physics, University of Exeter, Stocker Road, Exeter, EX4 4QL, United Kingdom \\ J. R. Childress and J. A. Katine \\ Hitachi Global Storage Technologies, San Jose Research Center, 650 Harry Road, San Jose, California 95120, USA
}

(Received 26 April 2005; published 27 June 2005)

\begin{abstract}
The picosecond magnetization dynamics of arrays of square $\mathrm{Ni}_{88} \mathrm{Fe}_{12} / \mathrm{Co}_{80} \mathrm{Fe}_{20}$ bilayer nanoelements were studied by optical pump-probe measurements. Experimentally observed modes were found to fall upon two branches, with a crossover from the high- to low-frequency regime as the element size was reduced to less than $220 \mathrm{~nm}$. Micromagnetic simulations revealed that the branches are associated with center and edge modes. The edge mode is found to dominate as the element size is reduced so that the magnetic response to a pulsed field becomes less spatially uniform.
\end{abstract}

DOI: 10.1103/PhysRevB.71.220409

PACS number(s): 75.40.Gb, 75.30.Ds, 75.50.Ss, 78.47.+p

Increased storage densities in magnetic data storage technology require the use of nanoscale magnetic elements. ${ }^{1} \mathrm{~A}$ full understanding of the spin-wave (SW) spectrum is also essential if higher data rates are to be achieved, for example, by the implementation of ultrafast precessional switching. ${ }^{2}$ Spatial confinement leads to quantized modes with frequency and spatial character that have a complicated dependence upon the exchange interaction and nonuniform demagnetizing field within the element. ${ }^{3-8}$ Interest in switching processes has led to the development of experimental techniques that allow SW excitations to be observed within the time domain. ${ }^{9,10}$ Although a number of studies have been performed upon microscale elements, nanoscale elements have not yet been extensively explored. Continued progress therefore requires the study of high-quality arrays of elements of identical shape and size. ${ }^{3,5,8,11}$

In this paper, time-resolved scanning Kerr microscopy (TRSKM) measurements ${ }^{9}$ are used to investigate magnetization dynamics in arrays of magnetic elements with size ranging from 64 to $630 \mathrm{~nm}$. Specifically, we study elements with a composition similar to that used in the free layer of a spin or tunnel valve recording sensor or a magnetic random access memory element. Using the TRSKM as a probe of the magnetization dynamics at the center of an array, we record its time-dependent response to a pulsed magnetic field. We show that the measured precession undergoes a crossover to a lower-frequency regime as the element size is reduced below a certain value. Numerical simulations performed with the object oriented micromagnetic framework (OOMMF) (Ref. 12) reproduce the observed variations in the mode frequencies and lead to the surprising conclusion that the magnetic response of the smallest nanoscale elements is less, rather than more, spatially uniform.

A $\mathrm{Ta}(50 \AA) / \mathrm{Co}_{80} \mathrm{Fe}_{20}(10 \AA) / \mathrm{Ni}_{88} \mathrm{Fe}_{12}(27 \AA) / \mathrm{Ta}(100 \AA)$ film was sputtered onto a Si substrate and patterned, using a combination of electron-beam lithography and ion milling, into square arrays of square elements. The element lengths (edge-to-edge separations) were 630 (37.5), 425 (21.9), 220 (95), 120 (37.5), and $64(48.4) \mathrm{nm}$, while the length of each array was about $4 \mu \mathrm{m}$. Scanning electron microscope images ${ }^{13}$ showed that the corners of the 64 and $120 \mathrm{~nm}$ elements were slightly rounded. The compositions of the $\mathrm{Ni}_{88} \mathrm{Fe}_{12}$ and $\mathrm{Co}_{80} \mathrm{Fe}_{20}$ alloys were chosen so that the magnetostriction of these two layers would partially compensate each other, leaving the bilayer with a small net negative magnetostriction. The $\mathrm{Co}_{80} \mathrm{Fe}_{20}$ alloy composition also gave a stable fcc structure. The direction of the easy axis (EA) was set by field annealing. Static magnetometry measurements made upon sheet samples of different thickness showed that $12.1 \AA$ of the $\mathrm{Ni}_{88} \mathrm{Fe}_{12}$ layer thickness was lost due to interdiffusion with the Ta cap. To make pump-probe measurements, a transmission line (TL) structure with a $30 \mu \mathrm{m}$ track width and separation was deposited around the elements so that they experienced an out-of-plane pulsed magnetic field. The measurements were performed in the TRSKM configuration described in detail elsewhere. ${ }^{6}$ In this configuration, the measured signal was proportional to the polar Kerr rotation, and hence, to the out-of-plane component of the magnetization. Several elements were typically within the area probed by the microscope so that only their average response was measured. A static field $\mathbf{H}$ was applied in the plane of the sample, parallel to the tracks of the TL. Each value of the static field used was approached from a much greater value, typically 1-2 kOe, that was sufficient to cause saturation. The small film thickness of the magnetic layer and reduced areal coverage led to a weak transient Kerr response with an amplitude of just a few microdegrees.

From the static magnetometry measurements the magnetizations of the $\mathrm{Co}_{80} \mathrm{Fe}_{20}$ and $\mathrm{Ni}_{88} \mathrm{Fe}_{12}$ were deduced to be 1445 and $585 \mathrm{emu} / \mathrm{cm}^{3}$, respectively. Since the two layers are strongly coupled by the interlayer exchange interaction, we can treat them as a single layer with thickness equal to the sum of their individual thicknesses $(24.9 \AA)$ and with magnetization given by a volume weighted average of their saturation magnetizations $\left(930 \mathrm{emu} / \mathrm{cm}^{3}\right)$. Uniaxial and surface anisotropy parameters of $4140 \pm 330 \mathrm{erg} / \mathrm{cm}^{3}$ and $0.156 \pm 0.022 \mathrm{erg} / \mathrm{cm}^{2}$ were deduced from TR measurements made upon a codeposited $6 \mu \mathrm{m}$ element, assuming a value of 2.1 for the $g$ factor. ${ }^{14}$ The sample EA was found to be canted by about $10^{\circ}$ from the length of the tracks of the TL structure. 


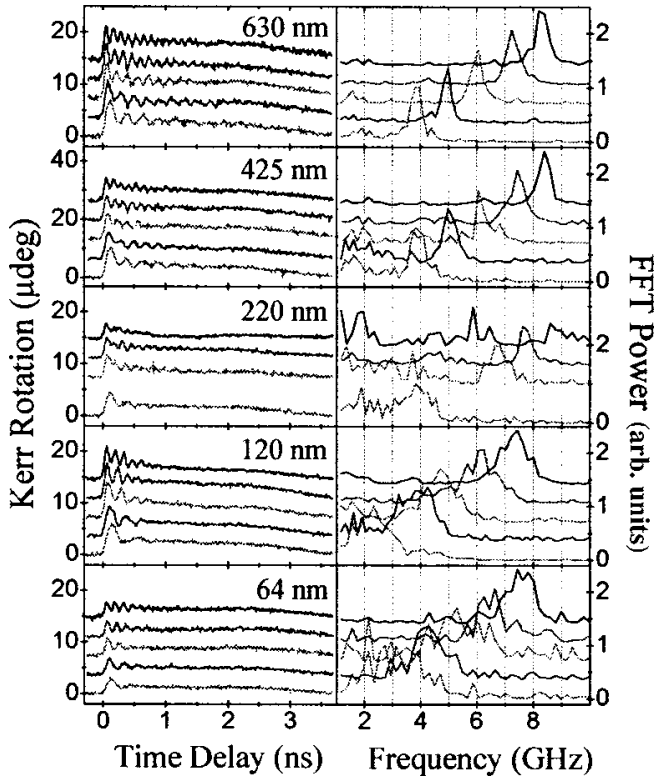

FIG. 1. (Color online) TR Kerr signals obtained from the element arrays and their FFT spectra are presented in the left and right columns, respectively. In each panel, curves from top to bottom correspond to bias field values of 772, 589, 405, 267, and 152 Oe, respectively; although for the $220 \mathrm{~nm}$ element there are no data for the field value of $267 \mathrm{Oe}$.

The measured time-resolved (TR) Kerr signals and their fast Fourier transform (FFT) spectra are presented in Fig. 1. The 630, 425, 120, and $64 \mathrm{~nm}$ element arrays showed a single mode. The TR signals from the array of $220 \mathrm{~nm}$ elements exhibit beating, and their FFT spectra suggest the presence of an additional mode at lower frequency, albeit of reduced relative intensity. The dependence of the frequency upon the element size is complicated and nonmonotonic. For the 630 and $425 \mathrm{~nm}$ element arrays, the frequency increases somewhat as the element size decreases. In the 120 and $64 \mathrm{~nm}$ element arrays, the same trend seems to hold, but the frequencies are reduced in comparison with those in the 630 and $425 \mathrm{~nm}$ element arrays. In the $220 \mathrm{~nm}$ element array, the higher-frequency modes continue the trend observed for the 630 and $425 \mathrm{~nm}$ element arrays, while the weaker lowfrequency modes continue the trend of the 120 and $64 \mathrm{~nm}$ element arrays. Hence, we observe two branches in the dependence of the frequency upon element size, with a crossover from one to the other at an element size close to $220 \mathrm{~nm}$. In the following, we refer to them as the "lower" and "higher" branch. The linewidth of the FFT peaks was noticeably greater in the 120 and $64 \mathrm{~nm}$ arrays in comparison with the 630 and $425 \mathrm{~nm}$ element arrays, which could be due to the greater imperfection of the smaller elements.

To identify the origin of the crossover, we simulated the magnetization dynamics within both single isolated elements and model arrays of $3 \times 3$ elements so that the center elements of these arrays had the same neighborhood as elements in the measured arrays. Simulations of the complete arrays were not possible due to the computation time required. Each array was divided into square cells with a height equal to the element thickness, while the lateral cell

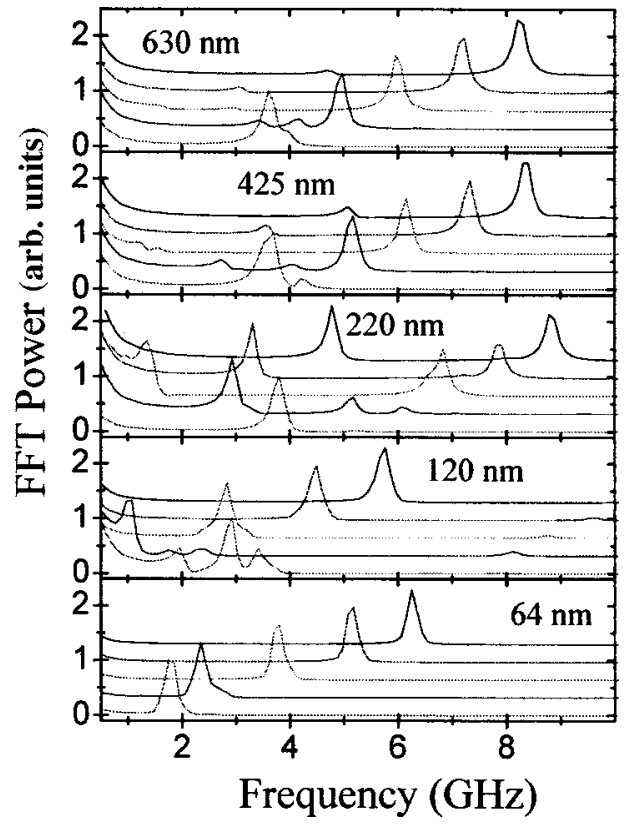

FIG. 2. (Color online) FFT spectra of the out-of-plane component of the average dynamic magnetization of the center element in the model arrays are shown for different values of the static field. In each panel, curves from top to bottom correspond to bias field values of 772, 589, 405, 267, and 152 Oe, respectively.

size was $7.5,6,5,4$, and $2.56 \mathrm{~nm}$ for $630,426,220,120$, and $64 \mathrm{~nm}$ element arrays, respectively. Additional simulations were performed with different cell sizes to ensure that the cell size did not influence the results.

The static state at each bias field value was prepared by allowing the magnetization to relax from a uniform state. At field values of 772,589 , and 405 Oe, the single elements of all sizes were found to be in the "flower" state. ${ }^{15}$ At a field value of $267 \mathrm{Oe}$, the 120, 220, and $425 \mathrm{~nm}$ elements switched to the "S" state, ${ }^{15}$ while the 64 and $630 \mathrm{~nm}$ elements remained in the flower state. At a field value of $152 \mathrm{Oe}$, all elements were in the $S$ state. The static state of the center elements in the model arrays was found to be similar in most cases. The exceptions were the 120 and $630 \mathrm{~nm}$ elements at a field value of 267 Oe that were in the flower and $S$ states, respectively. These static states were then used as the initial configuration in dynamical simulations, in which an out-of-plane pulsed field with 40 ps rise time, 2 ns decay time, and a magnitude of 15 Oe was applied to the sample, as in the experiment. The magnetic state was recorded every 5 ps during the first $8 \mathrm{~ns}$ after the excitation. Figure 2 shows the FFT spectra calculated from the out-ofplane component of the dynamic magnetization averaged over the volume of the central element of the different model arrays, while Fig. 3 compares the calculated frequencies of the dominant modes with experiment. The variation of the simulated spectra with element size is in striking agreement with experiment for the higher field values. In particular, the crossover from the higher- to the lower-frequency branch occurs at an element size of $220 \mathrm{~nm}$. Although several modes are generally present in the spectra, their relative amplitudes are different. In the $220 \mathrm{~nm}$ element, the higher and lower 


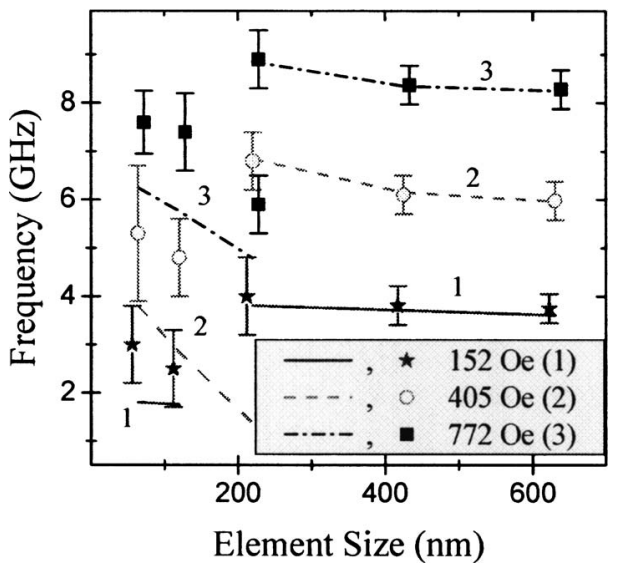

FIG. 3. (Color online) Extracted mode frequencies are plotted against element size for different bias fields. The symbols represent data points and the curves are simulations. A small horizontal shift has been applied to the experimental frequencies at different bias fields for clarity. The error bars represent the full width at half maximum of the spectral mode peaks.

branch modes have similar amplitudes. In contrast, a mode from either the higher or lower branch is dominant in the spectra obtained from the larger and smaller elements, respectively. The $64 \mathrm{~nm}$ element showed virtually single-mode response, with the peak amplitude of the other modes being more than three orders of magnitude smaller. At the lower field values, the behavior is less regular due to the increased dependence of the static configuration upon the interelement interaction and, in the experiment, upon pinning. The simulations also reproduce the experimental observation that the frequencies for a particular branch decrease monotonically with increasing element size and with decreasing static field. It was found that the simulations made with single isolated elements (not shown) and model element arrays were in very good agreement for all element sizes, although giving somewhat different frequencies for the lower branch mode at low field values.

Images of the dynamic magnetization within the elements allow us to explain the observed behavior in terms of the distribution of the static demagnetizing field within the elements, as shown in Fig. 4. The demagnetizing field is enhanced near the element edges that are perpendicular to the bias field. Hence, the magnitude of the total effective field, which is approximately equal to the difference of the applied and demagnetizing fields, is lower in the demagnetized regions than in the center, and so the magnetization precesses at different frequencies in these two regions, giving rise to the two observed modes. The crossover can be explained by the increase of the relative area of the demagnetized region, and hence the relative contribution of the edge mode, with reduction of the element size. At the same time the relative contribution of the higher-frequency mode decreases as the relative area in which it is localized is reduced. The reduction of the total effective field also favors the observation of the low-frequency modes, since the magnetization precession has greater amplitude at lower field.

The localized nature of the magnetization dynamics for the low-frequency mode also suggests that the discrepancy
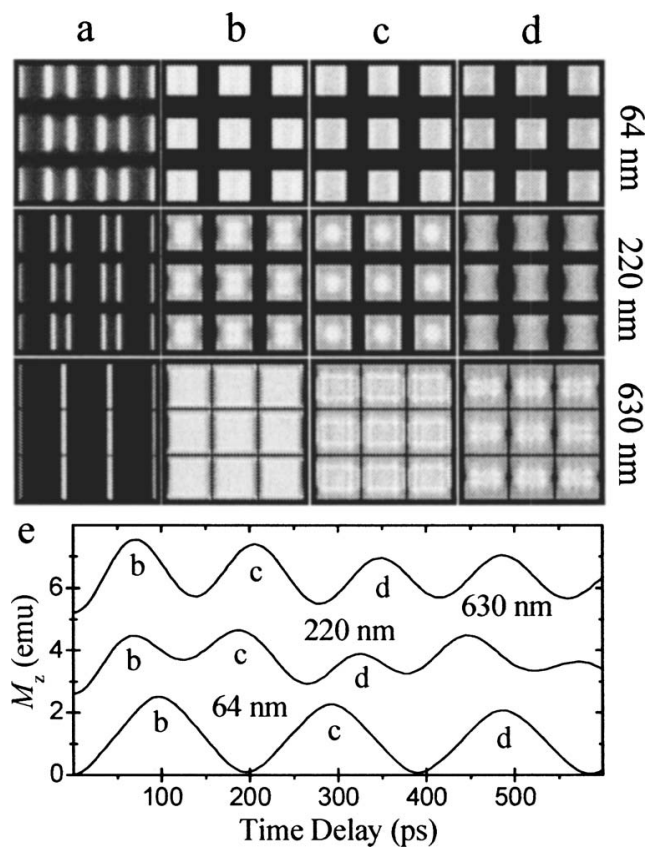

FIG. 4. The spatial distribution of the magnitude of the demagnetizing field in the model element arrays is shown in column (a) of the upper panel. White corresponds to the maximum of the demagnetizing field magnitude. Columns (b), (c), and (d) show the spatial distribution of the out-of-plane magnetization component at the marked antinodes in the TR average magnetization shown in panel (e). White corresponds to positive $M_{z}$. The static field of $589 \mathrm{Oe}$ was applied horizontally.

between simulated and measured frequency values is due to the exchange interaction. This is supported by the better agreement obtained for the larger elements and the highfrequency modes. Indeed, in the simulations, we assumed a value for the exchange stiffness appropriate for bulk Permalloy. Co-Fe alloys are known to have a larger exchange parameter. ${ }^{16}$ Hence, we performed a few simulations for a single isolated $220 \mathrm{~nm}$ element in which the exchange stiffness was increased by $50 \%$. The frequencies of the center and edge modes were found to increase by about $4 \%$ and $21 \%$, respectively. While this confirms our suggestion that the exchange is important, a systematic tuning of this and other magnetic parameters was not possible because of the computation time required.

While the simulations assumed a square element shape, the slight rounding of the 120 and $64 \mathrm{~nm}$ elements may affect the spatial distribution of the demagnetizing field. ${ }^{17}$ This may provide a simpler explanation of the observed discrepancy between experimental and simulated frequencies for these elements. Furthermore as the element size decreases, the fluctuations in the number of defects per element are expected to increase, and the importance of edge defects will be enhanced. The random nature of defects will cause different elements in an array to precess at different frequencies, and the frequency peak will be broadened, as observed in the 64 and $120 \mathrm{~nm}$ element arrays.

In summary, the magnetization dynamics of nanoscale magnetic elements were investigated by means of TR optical measurements and micromagnetic simulations. The dominant 
modes fell on two branches characterized by different frequencies. The simulations showed that the magnetization dynamics are nonuniform, and that the frequencies and relative amplitudes of the modes from the two branches are determined by the interplay between the exchange and demagnetizing fields. In particular, the dynamics become less uniform as the size of the element is reduced, and a mode confined by the demagnetizing field within the edge regions of the element becomes dominant. This nonuniformity may significantly contribute to noise in the read sensors of modern computer hard disk drives that are of size similar to the elements studied in the present work. Our results also apply to the ultrafast precessional switching of magnetic data storage elements induced by a pulsed magnetic field, and to the switching of nanoscale magnetic elements induced by a spinpolarized current.

The authors acknowledge the financial support of the UK Engineering and Physical Sciences Research Council (EPSRC), the New Energy and Industrial Technology Development Organization (NEDO), and the British Council.
*Email address: v.v.kruglyak@exeter.ac.uk

†Email address: r.j.hicken@exeter.ac.uk

${ }^{1}$ A. Moser, K. Takano, D. T. Margulies, M. Albrecht, Y. Sonobe, Y. Ikeda, S. H. Sun, and E. E. Fullerton, J. Phys. D 35, R157 (2002).

${ }^{2}$ W. K. Hiebert, G. E. Ballentine, and M. R. Freeman, Phys. Rev. B 65, 140404(R) (2002); T. Gerrits, H. A. M van den Berg, J. Hohlfeld, L. Bar, and T. Rasing, Nature (London) 418, 509 (2002).

${ }^{3}$ J. Jorzick, S. O. Demokritov, B. Hillebrands, M. Bailleul, C. Fermon, K. Y. Guslienko, A. N. Slavin, D. V. Berkov, and N. L. Gorn, Phys. Rev. Lett. 88, 047204 (2002); G. Gubbiotti, G. Carlotti, T. Okuno, T. Shinjo, F. Nizzoli, and R. Zivieri, Phys. Rev. B 68, 184409 (2003); G. Gubbiotti, M. Conti, G. Carlotti, P. Candeloro, E. Di Fabrizio, K. Y. Guslienko, A. Andre, C. Bayer, and A. N. Slavin, J. Phys.: Condens. Matter 16, 7709 (2004).

${ }^{4}$ C. Bayer, J. P. Park, H. Wang, M. Yan, C. E. Campbell, and P. A. Crowell, Phys. Rev. B 69, 134401 (2004); S. Tamaru, J. A. Bain, R. J. M. van de Veerdonk, T. M. Crawford, M. Covington, and M. H. Kryder, J. Appl. Phys. 91, 8034 (2002).

${ }^{5}$ T. M. Crawford, M. Covington, and G. J. Parker, Phys. Rev. B 67, 024411 (2003).

${ }^{6}$ R. J. Hicken, A. Barman, V. V. Kruglyak, and S. Ladak, J. Phys. D 36, 2183 (2003).

${ }^{7}$ A. Barman, V. V. Kruglyak, R. J. Hicken, J. M. Rowe, A. Kundrotaite, J. Scott, and M. Rahman, Phys. Rev. B 69, 174426 (2004); M. Belov, Z. Liu, R. D. Sydora, and M. R. Freeman, ibid. 69, 094414 (2004).

${ }^{8}$ G. N. Kakazei, P. E. Wigen, K. Y. Guslienko, V. Novosad, A. N. Slavin, V. O. Golub, N. A. Lesnik, and Y. Otani, Appl. Phys.
Lett. 85, 443 (2004).

${ }^{9}$ W. K. Hiebert, A. Stankiewicz, and M. R. Freeman, Phys. Rev. Lett. 79, 1134 (1997); A. Barman, V. V. Kruglyak, R. J. Hicken, A. Kundrotaite, and M. Rahman, Appl. Phys. Lett. 82, 3065 (2003); M. Buess, T. P. J. Knowles, R. Hollinger, T. Haug, U. Krey, D. Weiss, D. Pescia, M. R. Scheinfein, and C. H. Back, Phys. Rev. B 71, 104415 (2005).

${ }^{10}$ J. Vogel, W. Kuch, J. Camarero, K. Fukumoto, Y. Pennec, S. Pizzini, M. Bonfim, F. Petroff, A. Fontaine, and J. Kirschner, Phys. Rev. B 71, 060404(R) (2005); M. L. Schneider, A. B. Kos, and T. J. Silva, Appl. Phys. Lett. 85, 254 (2004); H. W. Schumacher, C. Chappert, T. Devolder, R. C. Sousa, and P. P. Freitas, J. Magn. Magn. Mater. 286, 362 (2005).

${ }^{11}$ S. Jung, B. Watkins, L. DeLong, J. B. Ketterson, and V. Chandrasekhar, Phys. Rev. B 66, 132401 (2002); Y. Zhai, J. Shi, X. Y. Zhang, L. Shi, Y. X. Xu, H. B. Haung, Z. H. Lu, and H. R. Zhai, J. Phys.: Condens. Matter 14, 7865 (2002).

${ }^{12}$ M. Donahue and D. G. Porter, OOMMF User's guide, Version 1.0, NIST Interagency Report No. NISTIR 6376, 1999 (unpublished) (URL: http://math.nist.gov/oommf).

${ }^{13}$ See EPAPS Document No. E-PRBMDO-71-R15522 for the images of the 425 and $64 \mathrm{~nm}$ elements. This document can be reached via a direct link in the online article's HTML reference section or via the EPAPS homepage (http://www.aip.org/ pubservs/epaps.html).

${ }^{14}$ V. V. Kruglyak, A. Barman, R. J. Hicken, J. R. Childress, and J. A. Katine, J. Appl. Phys. 97, 10A706 (2005).

${ }^{15}$ J. Miltat, G. Albuquerque, and A. Thiaville, Top. Appl. Phys. 83, 1 (2001).

${ }^{16}$ F. Schreiber and Z. Frait, Phys. Rev. B 54, 6473 (1996).

${ }^{17}$ A. Aharoni, J. Appl. Phys. 83, 3432 (1998). 